BENHA VETERINARY MEDICAL JOURNAL, VOL. 34, No. 3: 318-329, AUGUST 2018 A SPECIAL ISSUE FOR THE $6^{\text {TH }}$ SCIENTIFIC INTERNATIONAL CONFERENCE

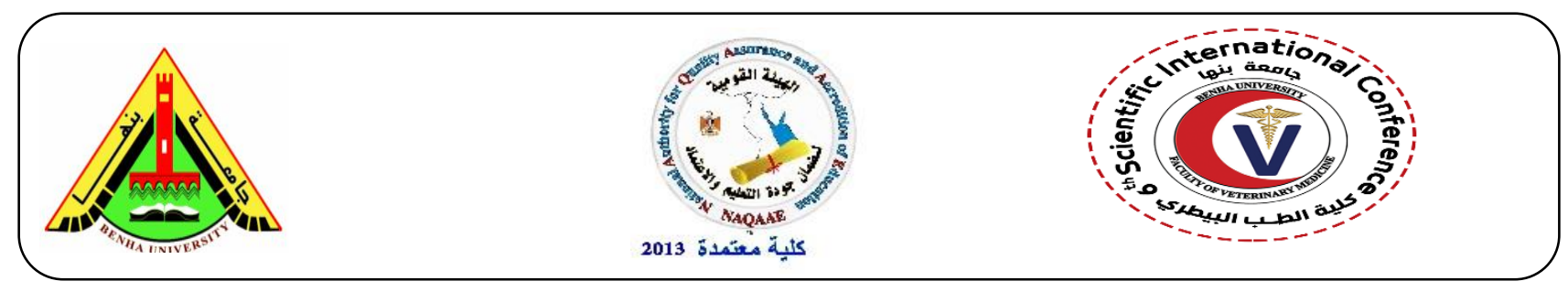

\title{
Pharmacokinetics and Tissue residues of Ceftiofur in normal and Aeromonas Hydrophilia Infected Catfish (Clarias lazera)
}

\author{
El-Sayed, M.G.A.*; Enas A. H. Farag** and Shimaa F. Elzoghby*** \\ * Department of pharmacology, Faculty of Veterinary Medicine, Benha University, Egypt. \\ ** Department of pharmacology, Animal Health Research Institute, Benha, Egypt. \\ ***Work at Vet. Hospital of Faculty of Veterinary Medicine, Benha University, Egypt.
}

\section{A B S T R A C T}

The pharmacokinetics of ceftiofur were studied in 78 catfish divided into 3 groups following IV and IM (single and repeated) administrations. Following a single IV injection of $5 \mathrm{mg} / \mathrm{kg}$ body weight of ceftiofur in normal catfish (Clarias lazera), serum concentration-time curve was best described by a two compartments open model with elimination half life $\left(\mathrm{t}_{0.5 \beta}\right)$, volume of distribution $\left(\mathrm{Vd}_{\mathrm{ss}}\right)$ and total body clearance $\left(\mathrm{CL}_{\mathrm{tot}}\right)$ of $5.700 \mathrm{~h}, 229.71 \mathrm{ml} / \mathrm{kg}$ and $0.642 \mathrm{ml} / \mathrm{kg} / \mathrm{min}$, respectively. Following a single IM administration of $5 \mathrm{mg}$ ceftiofur $/ \mathrm{kg}$ body weight in normal catfish (Clarias lazera), the peak serum concentration $\left(\mathrm{C}_{\max }\right)$ was $21.77 \mu \mathrm{g} / \mathrm{ml}$, achieved at a maximum time $\left(\mathrm{T}_{\max }\right)$ of $2.15 \mathrm{~h}$. The mean systemic bioavailability was $67.22 \%$. The serum concentrations of ceftiofur following repeated IM administration of $5 \mathrm{mg} / \mathrm{kg}$ body weight once daily for five consecutive days in healthy and experimentally Aeromonas hydrophilia infected catfish (Clarias lazera) showed a lower significant values recorded in experimentally Aeromonas hydrophilia infected catfish (Clarias lazera) than in normal ones. Ceftiofur showed accumulative behavior in serum of fish. Results of this study indicated that ceftiofur was useful for treatment of Aeromonas hydrophilia infections in fish. Ceftiofur was assayed in serum, liver, kidney, dorsal muscle, abdominal muscle and skin after 24, 48, 72,96 and $120 \mathrm{~h}$ from the last daily dose of $5 \mathrm{mg}$ ceftiofur/kg body weight for five days. . Ceftiofur could not be detected by spectrophotometer assay in all tested tissues in normal fish except in liver (four days), kidney (four days), and dorsal muscle (four days) post last administrations. Ceftiofur was completely cleared from serum and tissues at five days after the stoppage of drug dosage but in experimentally infected fish Ceftiofur could not be detected in all tested tissues except in liver (three days) and kidney (three days) post last administrations. Ceftiofur was completely cleared from all tissues at (four days) after the stoppage of drug dosage.

Key words: Pharmacokinetics Tissue residues Ceftiofur Aeromonas Hydrophilia

(http://www.bvmj.bu.edu.eg)

(BVMJ-34(3): 318-329, 2018

\section{Introduction}

Ceftiofur sodium (CEFTIOPHARCO) ${ }^{\circledR}$ is a chemotherapeutic agent is used in veterinary practice (Hornish et al.,2002) not only for large 
and small animals but also for poultry and fishes against Gram-positive and Gramnegative bacteria (Abd-Ellateif et al., 1998 and Fayaz et al.,2010) Ceftiofur sodium is one of the third generation cephalosporins. It is a broad spectrum antibiotic active against both Gram-positive and Gram-negative bacteria, including $\beta$-lactamase producing strains. It is bactericidal; destroying bacteria by preventing the synthesis of the cell wall (Yancey et al.,1987 and $\mathrm{Li}$ XL et al.,2011) The pharmacokinetics of ceftiofur has been investigated in many animal species including calves, chicken, pigs, foals, ducks, goats, elephants (Liu MC et al.,2010 , DoréElizabeth et al.,2011 and Adkesson MJ et al.,2012) respectively. Therefore, the aim of present work was undertaken to study the pharmacokinetic parameters of ceftiofur after IV and IM administration in normal and experimentally Aeromonas hydrophilia infected catfish (Clarias lazera). Also, the bioavailability of ceftiofur was calculated after IM administration in normal fish. Residues for ceftiofur in fish's tissues were studied in normal and Aeromonas hydrophilia infected catfish(Clarias lazera). .

\section{Material and Methods}

\section{Drug:}

Ceftiofur was used in this study under the trade name (Ceftiopharco®, sterile powder). Each vial contains ceftiofur sodium equivalent to $1 \mathrm{gm}$ ceftiofur. Each $\mathrm{ml}$ of reconstituted solution contains ceftiofur sodium equivalent to $50 \mathrm{mg}$ ceftiofur, which was manufactured by Pharco B International, Alexandria, Egypt.

Experimental fish:

Catfish (Clarias lazera) were used in this investigation. The weights of fish were $300 \pm 0.5$. Catfish (Claries lazera) were obtained from local farms in Benha city, El Qualubia government ,Egypt. It were feed on pelleted feed (25\% proteins, $8 \%$ fat) from ElAbassia farm, El- Sharkia governorate, Egypt twice daily.

Experimental design:

The fish were divided into 3 groups:-

Group (1): Group (1): It consist of 18 catfish (Calrias Lazera) divided into 6 glass aquaria. Each fish was injected IVly in the caudal vein with $5 \mathrm{mg}$ ceftiofur $/ \mathrm{kg}$ b.wt. These fish were left for 15 days after the IV injection to ensure complete excretion of ceftiofur from their bodies. Then the fish were injected IMly $5 \mathrm{mg}$ of ceftiofur $/ \mathrm{kg}$ b.wt in dorsal muscle to calculate bioavaibility of ceftiofur in normal catfish (ClariesLazera) .

Group (2): It consist of 30 catfish (Calrias lazera) divided into 10 glass aquaria. Each fish was injected IMly into dorsal muscle with 5 $\mathrm{mg}$ ceftiofur $/ \mathrm{kg}$ b.wt, once daily. Serum samples were taken and then IM dose was administered every $24 \mathrm{~h}$ for five consecutive days. Tissue samples were taken for assaying of drug residues after the last sampling.

Group (3): It included 30 catfish (Calrias lazera ) divided into 10 glass aquaria Twenty four $\mathrm{h}$ pure culture of chosen isolates of Aeromonas Hydrophilia was suspended in sterile saline using McFarland opacity tube number 3 ,bacterial suspension contain approximately $9 \times 10^{8}$ cell $/ \mathrm{ml}$. according to (Abd El Aziz et al.,1994).Each fish was inoculated with $0.2 \mathrm{ml}$ of bacterial suspension (each contain $9 \times 10^{8}$ cell $/ \mathrm{ml}$ ). The clinical symptoms as hemorrhage all over the skin especially at the base of the fins, erosion of the fins appeared after $48 \mathrm{~h}$ of injection with Aeromonas Hydrophilia suspension. Each fish was injected IMly with $5 \mathrm{mg}$ ceftiofur $/ \mathrm{kg}$ b. wt. every $24 \mathrm{~h}$ for five consecutive days. After that serum and tissue samples were taken for assaying of residues till disappearance of the drug from tissues.

Collection of samples:

Blood samples: 
Blood samples were collected from caudal vein following IV or IM administration in normal and experimentally infected cat fish. Blood samples are collected after 0.083, 0.167, $0.25,0.5,1,2,4,8,12$ and $24 \mathrm{~h}$ of administration in single study, and after 0.167 , $0.25,0.50,1,2,4,8,12$ and $24 \mathrm{~h}$ in the first day, second, third, fourth and fifth dose in the same study in repeated IM administration in normal and experimentally Aeromonas hydrophilia infected catfish (Clarias lazera). Serum samples were separated by centrifugation and stored until assay of ceftiofur.

Tissue samples:

Three fish were slaughtered, serum, Liver, kidney, muscle with skin (dorsal and abdominal muscles) were taken from fish after repeated IM injection in normal and experimentally Aeromonas hydrophilia infected catfish (Clarias lazera) for assaying of residues of ceftiofur at 24,48,72,96,120,186 h after the last sampling.

Analytical procedures:

Ceftiofur was assayed in serum by modified spectrophotometric method according to(Annapurna V et al.,2009). The standard solution of ceftiofur was prepared by dissolving $25 \mathrm{mg}$ ceftiofur in $25 \mathrm{ml}$ distilled water to obtain a concentration of $1000 \mu \mathrm{g} / \mathrm{ml}$. Standard concentrations were obtained by further dilution in distilled water and fish serum to obtain concentrations of $0.313,0.625$, $1.25,2.5,5, \quad 10,25$ and $50 \mu \mathrm{g} / \mathrm{ml}$ for preparation of standard curve of ceftiofur. The pharmacokinetic parameters were calculated by Winnonlin program, version 1.1 and other parameters according to (Baggot,1978a,b). All statistical analysis was carried out according to (Berly et al.,1990).

Assay of tissue samples:
Three milliliters of distilled water were added to one gram of the obtained tissue sample and homogenized in a porcelain morter by the aid of sterile sand. The homogenate was left in the refrigerator overnight then centrifuged. One milliliter of the supernatant was taken and subjected to the same procedures for assay in serum sample.

\section{Results}

Following a single IV injection of $5 \mathrm{mg}$ $/ \mathrm{kg}$ b.wt. in normal fish, ceftiofur could be detected therapeutically for $24 \mathrm{~h}$ post IV injection. The serum concentration - time curve of ceftiofur following IV injection showed that the drug obeyed two compartments open model. The disposition kinetics of ceftiofur following a single IV and IM administration were recorded in tables (1) and showed in figure (1). IM administration of $5 \mathrm{mg} / \mathrm{kg}$ b.wt every $24 \mathrm{~h}$ for five consecutive days in normal and Aeromonas hydrophilia infected catfish (Clarias lazera). revealed a lower significant serum ceftiofur concentration at all time sampling in Aeromonas hydrophilia infected catfish (Clarias lazera) than in normal fish. The pharmacokinetic parameters of ceftiofur after repeated IM administration in normal fish were compared to those in Aeromonas hydrophilia infected catfish (Clarias lazera) in table (2).

Tissue residues for ceftiofur in, liver, kidney, dorsal muscle and abdominal muscle with skin after repeated IM administration in normal fish were compared to those in Aeromonas hydrophilia infected catfish (Clarias lazera) were recorded in table (3). 


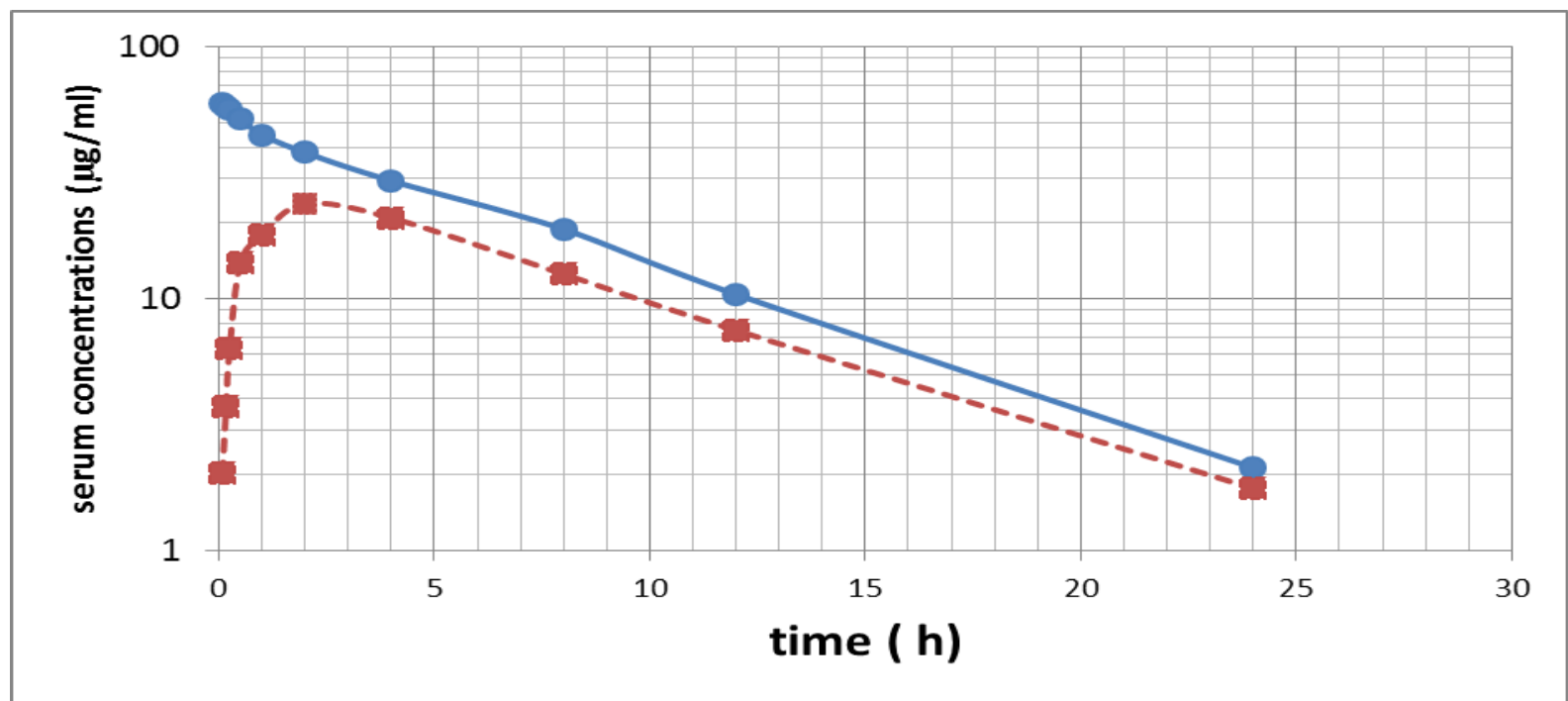

Figure (1): Arithmetic plot of serum of ceftiofur concentrations in normal catfish (Clarias lazera) following a single intramuscular injection of $5 \mathrm{mg} / \mathrm{kg}$ b.wt. in catfish previously given the same dose by a single intravenous injection $(n=6)$.

Table (1): Pharmacokinetic parameters of ceftiofur following a single IV and IM injection of $5 \mathrm{mg} / \mathrm{kg}$ b.wt. in normal fish $(n=6)$.

\begin{tabular}{cccc}
\hline Parameter $^{1}$ & $\mathrm{Unit}$ & $\mathrm{IV}$ & $\mathrm{IM}$ \\
\hline $\mathrm{B} . \mathrm{wt}$ & $\mathrm{Kg}$ & $1.900 \pm 0.053$ & $2.064 \pm 0.059$ \\
$\mathrm{C}^{\mathrm{o}}$ & $\mu \mathrm{g} / \mathrm{ml}$ & $58.11 \pm 0.684$ & - \\
$\mathrm{A}$ & $\mu \mathrm{g} / \mathrm{ml}$ & $16.489 \pm 0.338$ & $27.00 \pm 0.357$ \\
$\mathrm{~A}$ & $\mathrm{~h}^{-1}$ & $1.815 \pm 0.0364$ & - \\
$\mathrm{t}_{0.5(\alpha)}$ & $\mathrm{H}$ & $0.382 \pm 0.008$ & - \\
$\mathrm{K}_{\mathrm{ab}}$ & $\mathrm{h}^{-1}$ & - & $1.191 \pm 0.012$ \\
$\mathrm{t}_{0.5(\mathrm{ab})}$ & $\mathrm{H}$ & - & $0.581 \pm 0.006$ \\
$\mathrm{AUC}^{1}$ & $\mu \mathrm{g} / \mathrm{ml} / \mathrm{h}$ & $349.52 \pm 4.713$ & $334.60 \pm 2.031$ \\
$\mathrm{~V}^{1} \mathrm{c}$ & $\mathrm{ml} / \mathrm{kg}$ & $172.10 \pm 2.00$ & - \\
$\mathrm{V}_{\mathrm{d}(\mathrm{B})}$ & $\mathrm{ml} / \mathrm{kg}$ & $229.70 \pm 3.02$ & - \\
$\mathrm{V}_{\mathrm{dss}}$ & $\mathrm{ml} / \mathrm{kg}$ & $224.20 \pm 2.22$ & - \\
$\mathrm{K}_{\mathrm{el}}$ & $\mathrm{h}^{-1}$ & - & $0.119 \pm 0.001$ \\
$\mathrm{~K}_{12}$ & $\mathrm{~h}^{-1}$ & $0.435 \pm 0.012$ & - \\
$\mathrm{K}_{21}$ & $\mathrm{~h}^{-1}$ & $1.376 \pm 0.021$ & - \\
$\mathrm{B}$ & $\mu \mathrm{g} / \mathrm{ml}$ & $41.398 \pm 0.478$ & $29.226 \pm 0.203$ \\
$\mathrm{~B}$ & $\mathrm{~h}^{-1}$ & $0.121 \pm 0.002$ & - \\
$\mathrm{t}_{0.5(\beta)}$ & $\mathrm{h}$ & $5.700 \pm 0.077$ & - \\
$\mathrm{K}_{13}$ & $\mathrm{~h}^{-1}$ & $0.165 \pm 0.002$ & - \\
$\mathrm{C}_{\text {max }}$ & $\mu \mathrm{g} / \mathrm{ml}$ & - & $21.77 \pm 0.172$ \\
$\mathrm{~T}_{\max }$ & $\mathrm{h}$ & - & $0.121 \pm 0.001$ \\
$\mathrm{Cl}_{\text {tot }}$ & $\mathrm{ml} / \mathrm{kg} / \mathrm{min}$ & $0.642 \pm 0.008$ &
\end{tabular}


A \& B : Zero time plasma drug concentration intercepts of biphasic intravenous disposition curve. The coefficient $B$ is based on the terminal exponential phase $(\mu \mathrm{g} / \mathrm{ml}) . \alpha \& \beta$ : Hybrid rate constant of biphasic intravenous disposition curve values of $\alpha$ and $\beta$ are related to the slopes of distribution and elimination phase respectively, of biexponential drug disposition curve $\left(\mathrm{h}^{-1}\right)$.

AUC : Total area under the serum drug concentration versus time curve from $t=0$ to $t=\alpha$ after administration of a single dose.

$\mathrm{C}^{\circ} \quad$ : Drug concentration in the serum at zero time immediately after a single intravenous injection $(\mu \mathrm{g} / \mathrm{ml})$.

$\mathrm{C}_{\max }$ : Maximum serum concentration of drug in blood after extra vascular administration $(\mu \mathrm{g} / \mathrm{ml})$.

$\mathrm{C}_{\max }$ : Maximal serum concentration at steady - state during a multiple dose regimen $(\mu / \mathrm{ml})$.

$\mathrm{C}_{\min }$ : Minimal serum concentration at steady - state during a multiple dose regimen $(\mu \mathrm{g} / \mathrm{ml})$.

$\mathrm{Cl}_{\text {tot }}$ : The total clearance of a drug, which represents the sum of all clearance processes in the body $(\mathrm{ml} / \mathrm{kg} / \mathrm{min})$.

$\mathrm{K}_{\mathrm{ab}}$ : Apparent first order absorption rate constant $\left(\mathrm{h}^{-1}\right)$.

$\mathrm{K}_{\mathrm{el}}$ : First - order elimination rate constant for disappearance of drug from central compartment $\left(\mathrm{h}^{-1}\right)$.

$\mathrm{K}_{12}$ : First - order transfer rate constant for drug distribution from Central to

peripheral compartment $\left(\mathrm{h}^{-1)}\right.$.

$\mathrm{K}_{21}$ : First order transfer rate constant for drug distribution from Peripheral to central compartment $\left(\mathrm{h}^{-1}\right)$.

$\mathrm{t}_{0.5(\mathrm{ab})}$ : The absorption half- life (h).

$\mathrm{t}_{0.5(\alpha)}$ : Distribution half - life (h).

$\mathrm{t}_{0.5(\beta)}:$ Elimination half - life $(\mathrm{h})$.

$t_{\max }:$ The time at which the maximum concentration of drug was reached after extra vascular administration (h) .

$\mathrm{V} 1_{\mathrm{c}} \quad$ : The apparent volume of central compartment $(\mathrm{ml} / \mathrm{kg})$.

$\operatorname{Vd}_{(\mathrm{B})}:$ The apparent volume of distribution which calculated by extrapolation method $(\mathrm{ml} / \mathrm{kg})$.

$\mathrm{Vd}($ area) : The apparent volume of distribution which was calculated by the area method ( $\mathrm{ml} / \mathrm{kg})$.

$\mathrm{V}_{\mathrm{dss}}: \quad$ The apparent volume of distribution which was calculated by steady - state method $(\mathrm{ml} / \mathrm{kg})$. 
Table (2): Pharmacokinetic parameters of ceftiofur in normal (N) and experimentally Aeromonas hydrophilia infected catfish (Clarias lazera). (I) during repeated IM injections of $5 \mathrm{mg} / \mathrm{kg}$ b.wt. once daily for five consecutive days $(\mathrm{n}=3)$.

\begin{tabular}{|c|c|c|c|c|c|c|c|c|c|c|c|}
\hline \multirow[b]{2}{*}{$\begin{array}{c}\text { Paramete } \\
\text { r }\end{array}$} & \multirow[b]{2}{*}{ Unit } & \multicolumn{2}{|c|}{$1^{\text {st }}$ day } & \multicolumn{2}{|c|}{$2^{\text {nd }}$ day } & \multicolumn{2}{|c|}{$3^{\text {rd }}$ day } & \multicolumn{2}{|c|}{$4^{\text {th }}$ day } & \multicolumn{2}{|c|}{$5^{\text {th }}$ day } \\
\hline & & $\begin{array}{c}\mathrm{N} \\
(\mathrm{X} \pm \text { S.E. })\end{array}$ & $\begin{array}{c}\mathrm{I} \\
(\mathrm{X} \pm \text { S.E. })\end{array}$ & $\begin{array}{c}\mathrm{N} \\
(\mathrm{X} \pm \text { S.E. })\end{array}$ & $\begin{array}{c}\mathrm{I} \\
(\mathrm{X} \pm \text { S.E. })\end{array}$ & $\begin{array}{c}\mathrm{N} \\
(\mathrm{X} \pm \text { S.E. })\end{array}$ & $\begin{array}{c}\mathrm{I} \\
(\mathrm{X} \pm \text { S.E. })\end{array}$ & $\begin{array}{c}\mathrm{N} \\
(\mathrm{X} \pm \text { S.E. })\end{array}$ & $\begin{array}{c}\mathrm{I} \\
(\mathrm{X} \pm \mathrm{S} . \mathrm{E} .)\end{array}$ & $\begin{array}{c}\mathrm{N} \\
(\mathrm{X} \pm \text { S.E. })\end{array}$ & $\begin{array}{c}\text { I } \\
(\mathrm{X} \pm \text { S.E. })\end{array}$ \\
\hline $\mathrm{C}^{0}$ & $\mu \mathrm{g} / \mathrm{ml}$ & $\begin{array}{c}59.34 \pm 1.8 \\
4\end{array}$ & $61.94 \pm 1.55$ & $\underset{* * *}{63.32 \pm 1.84}$ & $\begin{array}{c}56.84 \pm 1.31 \\
* * *\end{array}$ & $\begin{array}{c}55.90 \pm 1.90 \\
* * *\end{array}$ & $\underset{ }{57.85 \pm 1.45}$ & $\begin{array}{c}60.44 \pm 2.12 \\
* * *\end{array}$ & $\underset{* * *}{67.13 \pm 1.54}$ & $\underset{* * *}{65.61 \pm 1.97}$ & $\begin{array}{c}68.50 \pm 1.71 \\
* * * *\end{array}$ \\
\hline A & $\mu \mathrm{g} / \mathrm{ml}$ & $\begin{array}{c}28.92 \pm 0.9 \\
25\end{array}$ & $\begin{array}{c}30.07 \pm 0.63 \\
2\end{array}$ & $\begin{array}{c}31.09 \pm 0.99 \\
5^{\text {*** }}\end{array}$ & $\begin{array}{c}25.87 \pm 0.59 \\
5\end{array}$ & $\begin{array}{c}24.65 \pm 0.73 \\
9^{* * *}\end{array}$ & $\begin{array}{c}25.32 \pm 0.58 \\
2^{* * *}\end{array}$ & $\begin{array}{c}23.32 \pm 0.81 \\
6\end{array}$ & $\begin{array}{c}33.08 \pm 0.76 \\
1\end{array}$ & $\begin{array}{c}25.78 \pm 0.87 \\
6^{* * *}\end{array}$ & $\begin{array}{c}32.35 \pm 0.84 \\
1^{* * * *}\end{array}$ \\
\hline $\mathrm{K}_{\mathrm{ab}}$ & $\mathrm{h}^{-1}$ & $\begin{array}{c}1.23 \pm 0.03 \\
9\end{array}$ & $1.34 \pm 0.031$ & $\underset{* * *}{1.73 \pm 0.014}$ & $\begin{array}{c}1.60 \pm 0.037 \\
*\end{array}$ & $\underset{* * *}{2.04 \pm 0.063}$ & $\underset{* * *}{1.64 \pm 0.038}$ & $2.60 \pm 0.083$ & $2.21 \pm 0.052$ & $\underset{* * *}{2.86 \pm 0.094}$ & $\begin{array}{c}3.20 \pm 0.074 \\
* *\end{array}$ \\
\hline $\mathrm{t}_{0.5(\mathrm{ab})}$ & $\mathrm{h}$ & $\begin{array}{c}0.563 \pm 0.0 \\
20\end{array}$ & $\begin{array}{c}0.517 \pm 0.01 \\
4\end{array}$ & $\begin{array}{c}0.401 \pm 0.01 \\
2^{*}\end{array}$ & $\begin{array}{c}0.433 \pm 0.01 \\
0^{* * *}\end{array}$ & $\begin{array}{c}0.340 \pm 0.01 \\
0\end{array}$ & $\begin{array}{c}0.423 \pm 0.01 \\
1^{* *}\end{array}$ & $\begin{array}{c}0.267 \pm 0.01 \\
0^{* * *}\end{array}$ & $\begin{array}{c}0.314 \pm 0.00 \\
7^{*}\end{array}$ & $\begin{array}{c}0.243 \pm 0.00 \\
7^{* * *}\end{array}$ & $\begin{array}{c}0.217 \pm 0.00 \\
5^{* * *}\end{array}$ \\
\hline $\mathrm{t}_{\max }$ & $\mathrm{h}$ & $\begin{array}{c}2.24 \pm 0.08 \\
1\end{array}$ & $2.64 \pm 0.061$ & $\underset{* * *}{2.54 \pm 0.084}$ & $\underset{* * *}{2.95 \pm 0.068}$ & $\underset{* * *}{2.49 \pm 0.085}$ & $\underset{* * *}{3.10 \pm 0.074}$ & $\underset{* * *}{2.42 \pm 0.077}$ & $\underset{* * *}{3.26 \pm 0.078}$ & $\underset{* * *}{2.12 \pm 0.074}$ & $\begin{array}{c}3.14 \pm 0.072 \\
* * * *\end{array}$ \\
\hline $\mathrm{C}_{\max }$ & $\mathrm{ug} / \mathrm{ml}$ & $\begin{array}{c}22.67 \pm 0.8 \\
16\end{array}$ & $\begin{array}{c}21.56 \pm 0.53 \\
9\end{array}$ & $\begin{array}{c}25.74 \pm 0.95 \\
2^{* * *}\end{array}$ & $\begin{array}{c}25.07 \pm 0.60 \\
2^{* * *}\end{array}$ & $\begin{array}{c}27.86 \pm 0.80 \\
8^{* * *}\end{array}$ & $\begin{array}{c}29.37 \pm 0.67 \\
6^{* * * *}\end{array}$ & $\underset{* * *}{31.26 \pm 1.09}$ & $\begin{array}{c}35.04 \pm 0.84 \\
1^{* * *}\end{array}$ & $\underset{* * *}{35.98 \pm 1.22}$ & $\begin{array}{c}39.07 \pm 0.93 \\
8^{* * *}\end{array}$ \\
\hline B & $\mu \mathrm{g} / \mathrm{ml}$ & $\begin{array}{c}30.42 \pm 0.8 \\
16\end{array}$ & $\begin{array}{c}31.87 \pm 0.73 \\
3\end{array}$ & $\begin{array}{c}32.23 \pm 0.95 \\
2^{* * *}\end{array}$ & $\begin{array}{c}30.97 \pm 0.71 \\
2^{* * *}\end{array}$ & $\begin{array}{c}31.25 \pm 1.00 \\
* * *\end{array}$ & $\begin{array}{c}32.53 \pm 0.78 \\
1^{* * *}\end{array}$ & $37.12 \pm 1.11$ & $\begin{array}{c}34.05 \pm 0.81 \\
7^{* * *}\end{array}$ & $\begin{array}{c}39.83 \pm 1.27 \\
*\end{array}$ & $\begin{array}{c}63.15 \pm 0.83 \\
1^{* *}\end{array}$ \\
\hline $\mathrm{K}_{\mathrm{el}}$ & $\mathrm{h}^{-1}$ & $\begin{array}{c}0.129 \pm 0.0 \\
04\end{array}$ & $\begin{array}{c}0.379 \pm 0.00 \\
8\end{array}$ & $\begin{array}{c}0.106 \pm 0.00 \\
7^{* *}\end{array}$ & $\begin{array}{c}0.339 \pm 0.00 \\
8^{* * *}\end{array}$ & $\begin{array}{c}0.059 \pm 0.00 \\
2^{* * *}\end{array}$ & $\begin{array}{c}0.323 \pm 0.00 \\
7^{\text {*** }}\end{array}$ & $\begin{array}{c}0.051 \pm 0.00 \\
2^{* * *}\end{array}$ & $\begin{array}{c}0.316 \pm 0.00 \\
7^{* * *}\end{array}$ & $\begin{array}{c}0.044 \pm 0.00 \\
1^{\text {*** }}\end{array}$ & $\begin{array}{c}0.319 \pm 0.00 \\
8^{* * *}\end{array}$ \\
\hline $\mathrm{t}_{0.5(\mathrm{kel})}$ & $\mathrm{h}$ & $\begin{array}{c}5.37 \pm 0.19 \\
3\end{array}$ & $1.83 \pm 0.046$ & $\begin{array}{c}6.54 \pm 0.189 \\
*\end{array}$ & $\begin{array}{c}2.04 \pm 0.049 \\
*\end{array}$ & $\begin{array}{c}11.75 \pm 0.36 \\
4\end{array}$ & $\underset{* * *}{2.15 \pm 0.051}$ & $\begin{array}{c}13.39 \pm 0.45 \\
5^{* * *}\end{array}$ & $\underset{* * *}{2.19 \pm 0.053}$ & $\begin{array}{c}15.60 \pm 0.53 \\
0\end{array}$ & $\begin{array}{c}2.17 \pm 0.054 \\
* * * *\end{array}$ \\
\hline $\mathrm{Cl}_{\text {tot }}$ & $\begin{array}{c}\mathrm{ml} / \mathrm{kg} / \\
\mathrm{min}\end{array}$ & $\begin{array}{c}0.0004 \pm 0 . \\
00001\end{array}$ & $\begin{array}{c}0.0005 \pm 0.0 \\
001\end{array}$ & $\begin{array}{c}0.0003 \pm 0.0 \\
0001^{* * *}\end{array}$ & $\begin{array}{c}0.0005 \pm 0.0 \\
0001^{* *}\end{array}$ & $\begin{array}{c}0.0002 \pm 0.0 \\
0004^{* * *}\end{array}$ & $\begin{array}{c}0.0005 \pm 0.0 \\
0001^{* * *}\end{array}$ & $\begin{array}{c}0.00001 \pm 0 \\
00002^{* * *}\end{array}$ & $\begin{array}{c}0.0004 \pm 0.0 \\
0001^{\text {*** }}\end{array}$ & $\begin{array}{c}0.00009 \pm 0 \\
00003^{* * *}\end{array}$ & $\begin{array}{c}0.0004 \pm 0.0 \\
0001^{* * *}\end{array}$ \\
\hline
\end{tabular}

$(*)$ : Represent the significance in comparison with data of normal group. $* \mathrm{P}<0.05-* * \mathrm{P}<0.01 \quad$ _*** $\mathrm{P}<0.001$ 
Table (3): Serum $(\mu \mathrm{g} / \mathrm{ml})$ and tissue $(\mu \mathrm{g} / \mathrm{g})$ concentrations of ceftiofur $(\mu \mathrm{g} / \mathrm{ml})$ in normal $(\mathrm{N})$ and experimentally Aeromonas hydrophilia infected catfish (Clarias lazera) (I) during repeated IM injections of $5 \mathrm{mg} / \mathrm{kg}$ b.wt. once daily for five consecutive days $((\mathrm{n}=3)$.).

\begin{tabular}{|c|c|c|c|c|c|c|c|c|c|c|}
\hline Time & \multicolumn{2}{|c|}{ After first day } & \multicolumn{2}{|c|}{ After second day } & \multicolumn{2}{|c|}{ After third day } & \multicolumn{2}{|c|}{ After forth day } & \multicolumn{2}{|c|}{ After fifth day } \\
\hline Tissue & $\begin{array}{c}\mathrm{N} \\
(\overline{\boldsymbol{X}} \pm \text { S.E. })\end{array}$ & $\begin{array}{c}\mathrm{I} \\
(\overline{\boldsymbol{X}} \pm \text { S.E. })\end{array}$ & $\begin{array}{c}\mathrm{N} \\
(\overline{\boldsymbol{X}} \pm \text { S.E. })\end{array}$ & $\begin{array}{c}\mathrm{I} \\
(\overline{\boldsymbol{X}} \pm \text { S.E. })\end{array}$ & $\begin{array}{c}\mathrm{N} \\
(\overline{\boldsymbol{X}} \pm \text { S.E. })\end{array}$ & $\begin{array}{c}\mathrm{I} \\
(\overline{\boldsymbol{X}} \pm \text { S.E. })\end{array}$ & $\begin{array}{c}\mathrm{N} \\
(\overline{\boldsymbol{X}} \pm \text { S.E. })\end{array}$ & 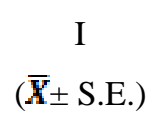 & $\begin{array}{c}\mathrm{N} \\
(\overline{\boldsymbol{X}} \pm \text { S.E. })\end{array}$ & $\begin{array}{c}\text { I } \\
\left(\overline{X_{ \pm}} \text {S.E. }\right)\end{array}$ \\
\hline Serum & $13.65 \pm 0.372$ & $10.830 \pm .662$ & $1.083 \pm 0.0124$ & - & - & - & - & - & - & - \\
\hline Liver & $33.68 \pm 0.110$ & $12.633 \pm 1.26$ & $16.173 \pm 0.191$ & $\begin{array}{c}4.546 \pm \\
0.329\end{array}$ & - & $0.289 \pm 0.072$ & $0.865 \pm 0.070$ & - & - & - \\
\hline Kidney & $35.74 \pm 0.248$ & $\begin{array}{c}17.688 \pm \\
0.996\end{array}$ & $22.09 \pm 0.435$ & $\begin{array}{l}7.363 \pm \\
0.449\end{array}$ & $7.796 \pm 0.124$ & $0.649 \pm 0.0135$ & $1.37 \pm 0.190$ & - & - & - \\
\hline $\begin{array}{l}\text { Dorsal } \\
\text { muscle }\end{array}$ & $26.86 \pm 0.330$ & $8.230 \pm 0.375$ & $15.886 \pm 0.191$ & $865 \pm 0.124$ & $10.106 \pm 0.073$ & - & $0.732 \pm 0.067$ & - & - & - \\
\hline $\begin{array}{c}\text { abdominal } \\
\text { muscle }\end{array}$ & $19.64 \pm 0.190$ & $4.043 \pm 0.591$ & $7.146 \pm 0.329$ & - & $6.356 \pm 0.286$ & - & - & - & - & - \\
\hline
\end{tabular}

(*): Represent the significance in comparison with data of normal group.
$* * \mathrm{P}<0.01$
$* * * \mathrm{P}<0.001$ 


\section{Discussion}

In the present investigation, IV injection of $5 \mathrm{mg}$ ceftiofur / $\mathrm{kg}$ b.wt. in normal catfish(Clarias lazera), showed that the drug disposition best fitted a two-compartments open model, compartment of plasma and rapid equilibrating tissues, and a deeper slower compartment. The obtained result was consistent with those reported for ceftiofur in ducks (Liu et al.,2010) in neonatal foals ( Hall ,2011) and for cefquinome in chicken( Xie ,2013).

The $\mathrm{Vd}_{\mathrm{ss}}$ is a clearance-independent volume of distribution that is used to calculate the drug amount in the body under equilibrium conditions ( Toutain et al.,2004) The $\mathrm{Vd}_{\mathrm{ss}}$ for ceftiofur was $224.20 \mathrm{ml} / \mathrm{kg}$, suggesting limited penetration through biological membranes and tissue distribution after IV administration in catfish(Clarias lazera). The obtained result agreed with the data reported after IV administration of ceftiofur in calves $(0.200$ $\mathrm{L} / \mathrm{kg}$ ) by (Whittem et al.,1995). On the other hand, volume of distribution was smaller than those recorded in calves $(0.284 \mathrm{~L} / \mathrm{kg}$ ) (Brown et al.,1996), in goats $(0.25 \mathrm{~L} / \mathrm{kg}$ ) (Courtin et al., 1997) in foals $(0.83 \mathrm{~L} / \mathrm{kg})$ (Meyer et al.,2009) and in ducks $(0.48 \mathrm{~L} / \mathrm{kg}$ ) (Xu et al.,2012).These differences might be due to the specific interspecies variations.

Ceftiofur was transferred from central to peripheral compartment at a slower rate $\left(\mathrm{K}_{12}=\right.$ $0.435 \mathrm{~h}^{-1}$ ) than its passage from peripheral compartment to central compartment $\left(\mathrm{K}_{21}=\right.$ $\left.1.376 \mathrm{~h}^{-1}\right)$. These values were nearly similar to that reported for ceftiofur in cows $\left(\mathrm{K}_{12}=0.473\right.$ $\left.\mathrm{h}^{-1}\right)$ and $\left(\mathrm{K}_{21}=0.950 \mathrm{~h}^{1}\right)$ by (Tohamy , 2008).

The elimination half-life $\left(\mathrm{t}_{0.5(\beta)}\right)$ of ceftiofur following a single IV injection was equal to $5.700 \mathrm{~h}$. This observation agreed with the data reported after IV administration of ceftiofur in cows and calves $\left(\mathrm{t}_{0.5(\beta)}\right)=5.09,5.05 \mathrm{~h}$ by $(\mathrm{El}-$ Gendy 2007 and Tohamy, 2008), respectively.
On contrast, this obtained value was longer than those recorded in other species as chickens (4.23 h)( Amer et al.,1998) camels ( 3.18 ) (Goudah,2007) and ducks (2.28, $3.64 \mathrm{~h}$ )( Liu et al.,2010 and $\mathrm{Xu}$ et al.,2012) On the other hand, it was shorter than those showed in cattle $(7.12 \mathrm{~h}$ ) (Whittem et al.,1995) and in foals $(7.8 \mathrm{~h})$ (Meyer et al.,2009).

The rate of total body clearance $\left(\mathrm{CL}_{\mathrm{tot}}\right)$ of ceftiofur following IV injection was $0.642 \pm 0.008 \mathrm{ml} / \mathrm{kg} / \mathrm{min}$.

Following a single IM administration of 5 mg ceftiofur / $\mathrm{kg}$ b.wt, the drug reached its maximum serum concentrations after $2 \mathrm{~h}$ of administration $(22.01 \mu \mathrm{g} / \mathrm{ml})$. Ceftiofur could be detected in serum in a therapeutic level $(1.85 \mu \mathrm{g} / \mathrm{ml})$ at $24 \mathrm{~h}$. The mean peak serum concentrations of ceftiofur $\left(\mathrm{C}_{\max }\right)$ was $(21.771 \mu \mathrm{g} / \mathrm{ml})$ achieved at maximum time $\left(\mathrm{t}_{\max }\right.$ $=2.15 \mathrm{~h}$ ). These values were similar to those recorded for ceftiofur in chickens $(27.83 \mu \mathrm{g} / \mathrm{ml}$ at $2.39 \mathrm{~h})$

(Amer et al.,1998). On contrast, the obtained results were different from those reported in in camels $(10.34 \mu \mathrm{g} / \mathrm{ml}$ at $1.22 \mu$ )(Goudah ,2007)., in cows $(7.83 \mu \mathrm{g} / \mathrm{ml}$ at $1.55 \mathrm{~h})($ Tohamy ,2008)., in ball python $(7.09 \mu \mathrm{g} / \mathrm{ml}$ at $2.17 \mathrm{~h}$ )(Adkesson et al.,2011)

in guinea fowl $(5.26 \mu \mathrm{g} / \mathrm{ml}$ at $19.3 \mathrm{~h})($ Wojick et al.,2011), in ducks ( $6.44 \mu \mathrm{g} / \mathrm{ml}$ at $0.49 \mathrm{~h})$ ( $\mathrm{Xu}$ et al., in chickens $(3.04 \mu \mathrm{g} / \mathrm{ml})(\mathrm{Xie}$ et al.,2013) and in pigs $(28.3 \mu \mathrm{g} / \mathrm{ml}$ at $2 \mathrm{~h})$.( Brown et al.,1999)These variations might be attributed to anatomical differences between species, healthy status and the dose administered in each case (El-Sayed et al., 1989).

The bioavailability of ceftiofur in normal fish was $(67.22 \%)$. This value referred to a good absorption of ceftiofur from its site of IM administration. This value was close to those recorded for ceftiofur in camels $(68.70 \%)$ (Goudah ,2007) and in ducks(79.25\%)( Xu et 
al.,2012). On contrast, this value was lower than that reported for ceftiofur in horses $(100 \%)$ ( Collard et al.,2011) and for cefquinome in ducks $(93.28 \%)($ Yuan et al.,2011)and in chickens $(95.81 \%)(\mathrm{Xie}$ et al.,2013).

The obtained blood levels of ceftiofur in experimentally Aeromonas hydrophilia infected catfish (Clarias lazera) were significantly decreased than those in normal fish following repeated IM administrations. These lower blood concentrations in infected fish might be attributed to the higher penetrating power of ceftiofur to the diseased tissues(Baggot, 1980). The relative higher serum concentrations of ceftiofur after the last dose compared to the first doses indicated the accumulation of ceftiofur in blood during multiple dosing at $24 \mathrm{~h}$ intervals for five consecutive days. These observations agreed with data reported by(El-Banna et al.,1998), who found that progressive daily increase in the mean serum concentrations following the IM injection of ciprofloxacin in lactating goats in a daily dose of $5 \mathrm{mg} / \mathrm{kg} \mathrm{b}$. wt. for five consecutive days and (Credille et al.,2012), who recorded a progressive daily increase in mean concentration in blood following IM injection of ceftiofur in weanling foals at a daily dose of $6.6 \mathrm{mg} / \mathrm{kg}$ b.wt. for four consecutive days. The obtained result was inconsistent with that reported by(Brown et al.,1990) who found a little drug accumulation after multiple dosing of norfloxacin in dogs.

Repeated IM administration of $5 \mathrm{mg}$ ceftiofur $/ \mathrm{kg}$ b.wt every $24 \mathrm{~h}$ for five consecutive days in normal and experimentally Aeromonas hydrophilia infected catfish (Clarias lazera) were revealed that the drug could only be detected in blood, muscle and skin till $72 \mathrm{~h}$ post last dose and till $96 \mathrm{~h}$ post last administration in liver, kidney and dorsal muscle. The high clearance of ceftiofur indicated the reduced possibility of finding residues of ceftiofur in fish after treatment and shorter withdrawal time for this antimicrobial (five days). Results showed that kidney and liver contained the highest drug concentrations (35.74, $33.68 \mu \mathrm{g} / \mathrm{g}$ respectively), while the lowest drug concentrations was found in abdominal muscle with skin $(19.64 \mu \mathrm{g} / \mathrm{g}), 24 \mathrm{~h}$ after the stoppage of drug medication. This result slightly agreed with that recorded for ceftiofur in swines that reported by ( $\mathrm{Li}$ et al.,2013), who found that the highest concentration in kidney $(2.589 \mu \mathrm{g} / \mathrm{g})$ at $12 \mathrm{~h}$, while the concentration of ceftiofur in all of tissues at three days was lower than the Maximum Residue Limit (MRL).

\section{Conclusion}

The IM bioavailability of ceftiofur is excellent, so it is recommended to be used against enteric and systemic Aeromonas hydrophilia infected catfish (Clarias lazera) infection. Repeated IM administrations of 5 $\mathrm{mg} / \mathrm{kg}$ b.wt. ceftiofur once daily for five consecutive days would provide an effective concentration against Aeromonas hydrophilia in infected catfish (Clarias lazera). Treated fish must not be slaughtered before 5 days from last dose of repeated administration of ceftiofur to withdraw the drug residues from all tissues of treated fish.

\section{References}
Abd El Aziz, E.S (1994) Immunological studies on Aeromonas and pseudomonas bacterial infection in fresh water fishes. $\mathrm{Ph}, \mathrm{D}$ Thesis, Cairo Univ, Egypt .

Abd-Ellateif AE and El-Din IMG (1998). The role of ceftiofur sodium (Excenel) in the control of pasteurella multocida infection in chickens. Proceeding of the 
4th Veterinary Medicine Zagazig Congress, Hurghada, 26- 28 : 632-645.

Adkesson MJ, Fernandez VE, Cox $\mathrm{S}$ and Martín JT (2011).Pharmacokinetics of a long-acting ceftiofur formulation (ceftiofur crystalline free acid) in the ball python (Python regius). J. Zoo Wildl. Med., 42 (3) : 444-450.

Adkesson MJ, Junge RE, Allender MC and Martín JT (2012).Pharmacokinetics of a long-acting ceftiofur crystalline-free acid formulation in Asian elephants (Elephas maximus) . Am. J. Vet. Res., 73 (10): 1512-1518.

Albarellos GA, Kreil VE and Landoni MF(2007).Pharmacokinetics of ceftriaxone after IV, IM and subcutaneous administration to domestic cats. J. Vet. Pharmacol. Ther., 30(4):345-352.

Amer AM, Fahim EM and Ibrahim RK (1998).Effect of aflatoxicosis on the kinetic behaviour of ceftiofur in chickens. Res. Vet. Sci., 65 (2): 115118.

Angkana T, Walaisiri Y, Pornpen WG and Dahrit N (2008).Pharmacokinetics of ceftiofur hydrochloride in pigs infected with porcine reproductive and respiratory syndrome virus. J. Antimicrob. Chemotherap., 369-373.

Annapurna V, Jyothi G, Rambabu C and Sailaja BB (2009). Specrophotometric determination of ceftiofur hydrochloride using nbromosuccinimide and $\mathrm{p}$ dimethylaminobenzaldehyde. J. Chem., 6 (3): 763-769.

Baggot JD ( 1978a,b). Some aspect of clinical pharmacokinetics in veterinary medicine II . J. Vet. Pharm. Ther, 1: 518.111-118.

Baggot JD (1980). Distribution of antimicrobial agents in normal and diseased animals. J.A.V.M.A., 19(76):1085-1090.

Berly DA and Lindgren BW (1990)."Statistics: Theory and Methods". Brooks1 cole publishing company, Pacific Grove California.

Brown SA, Chester ST and Rob EJ (1996).Effects of age on the pharmacokinetics of single dose ceftiofur sodium administered IMly or IVly to cattle. J. Vet. Pharmacol. and Therap.,19 (1): 32-38.

Brown SA, Hanson BJ, Mignot A, Millérioux L, Hamlow PJ, Hubbard VL, Callahan JK and Kausche FM (1999).Comparison of plasma pharmacokinetics and bioavailability of ceftiofur sodium and ceftiofur hydrochloride in pigs after a single IM injection . J. Vet. Pharmacol. and Therap., 22 (1): 35-40.

Brown SA, Cooper J, Gouze JJ, Gerco DS, Weise DW and Buck JM (1990). Pharmacokinetics of norfloxacin in dogs after single IV and single and multiple oral administration of the drug . A.m.J.Vet.Res.,51:1065-1070 .

Collard WT, Cox SR, Lesman SP, Grover GS, Boucher JF, Hallberg JW, Robinson JA and Brown SA (2011).Pharmacokinetics of ceftiofur crystalline free acid sterile suspension in the equine. J. Vet. Pharmacol. and Therap.,1365-2885.

Courtin F, Craigmill A L, Wetzlich S E, Gustafson CR and Arndt TS (1997).Pharmacokinetics of ceftiofur and metabolites after single IV and IM administration and multiple IM administrations of ceftiofur sodium to dairy goats. J. Vet. Pharmacol. and Therap., 20 (5):368-373.

Credille BC, Giguère S, Berghaus LJ, Burton AJ, Sturgill TL, Grover GS, Donecker 
JM and Brown SA (2012). Plasma and pulmonary disposition of ceftiofur and its metabolites after IM administration of ceftiofur crystalline free acid in weanling foals. J. Vet. Pharmacol. and Therap., 35 (3): 259-264.

Deshmukh PV, Deore MD, Gatne MM, Ramesh and Bhoir PS(2007).Tissue concentrations of ceftiofur after single subcutaneous and oral administration in chicken. J. Bomb. Vet. Coll., 15 : 9798.

Doré-Elizabeth AJA, Rowe JD, Carlson JL, Wetzlich SE, Kieu HT and Tell LA (2011).Pharmacokinetics of ceftiofur crystalline free acid after single subcutaneous administration in lactating and nonlactating domestic goats (Capra aegagrus hircus). J. Vet. Pharmacol. and Therap., 34(1): 2530.1365-2885.

El-Banna HA and Abo-El-Sooud K (1998).Disposition kinetics of ciprofloxacin in lactating goats .Dtsch. Tierarztl. Wochenschr., 105:35-38.

El-Gendy AAM, Tohamy MA and Ismail M (2007).Comparative pharmacokinetic and renal clearance study of ceftiofur in cross bred and buffalo calves. BS. Vet. Med., 17: 69-77.

El-Sayed MG, Hatem ME and El-Komy AA (1989). Disposition kinetic of gentamicin in normal and endometric cow using microbiological assay. Dtsch. Tierarztl. Wochenschr, 96: 412415 .

Fayaz A M , Balaji K, Girilal M, Yadav R, Kalaichelvan $\mathrm{P} T$ and Venketesan $\mathrm{R}$ (2010). Biogenic Synthesis of Silver Nanoparticles and Their Synergestic Effect with Antibiotics: A Study against gram positive and gram negative bacteria. Nanomedicine:
Nanotechnology, Biology and Medicine, 6 (1) : 103-109.

Goudah A (2007). Pharmacokinetics of ceftiofur after single IV and IM administration in camels (Camelus dromedarius). J. Vet. Pharmacol. Therap., 4: 371-374.

Hall TL, Tell LA, Wetzlich SE, McCormick JD, Fowler LW and Pusterla N (2011).Pharmacokinetics of ceftiofur sodium and ceftiofur crystalline free acid in neonatal foals. J. Vet. Pharmacol. and Therap., 34 (4): 403409.1365-2885.

Halstead SL, Walker RD, Baker JC, Holland RE, Gstein GE and Hauptman JG(1992).Pharmacokinetic evaluation of ceftiofur in serum, tissue chamber fluid and bronchial secretions from healthy beef-bred calves. Can .J. Vet. Res., 56 (4): 269-274.

Hornish R.E. and Kotarski S.F. (2002).Cephalosporins in veterinary medicine, ceftiofur use in food animals. Curr. Top. Med. Chem., 2 (7): 717-731.

Li SP, Huang XH, Kong XK, Zhang XH and Li XY (2013). Determination of ceftiofur residues in swine tissues. Acta Veterinaria Et Zootechnica Sinica., 44(8): 1311-1316.

Li XL, Weizhen M, Michael L, Machesk Y, Yates SR and Katterhenry M (2011). Degradation kinetics and mechanism of antibiotic ceftiofur in recycled water derived from a beef farm. J. Agri. Food Chem., 59(18): 10176-10181.

Liu MC, Jiao Y, Li J, Song FJ and Ding $\mathrm{F}(2010)$.Studies on blood pharmacokinetics and bioavailability of ceftiofur in Ducks. J. Shenyang Agri. Uni., 41(3): 346-349.

Meyer S, Giguère S, Rodriguez R, Zielinski RJ, Grover GS and Brown SA 
(2009).Pharmacokinetics of IV ceftiofur sodium and concentration in body fluids of foals. J. Vet. Pharmacol. and Therap., 32 (4): 309-16.1365-2885.

Tohamy MA (2008).Pharmacokinetics of ceftiofur sodium administered concomitantly with dipyrone in healthy and feverish cows. J. Egypt Soc. Pharmacol. Exp. Ther., 29: 539-550.

Toutain PL and Bousquet-Melou A (2004).Volumes of distribution. J. Vet. Pharmacol. Therap., 27: 441-453.

Whittem T, Freeman DA, Hanlon D and Parton K (1995). The effect on the pharmacokinetics of IV ceftiofur sodium in dairy cattle of simultaneous IV acetyl salicylic acid (aspirin) or probenecid. J.Vet. Pharmacol. Therap., 18: 61-67.

Wojick BK,Jennifer N L, Michael JA, Sherry KC and Kathryn CG (2011).Pharmacokinetics of long-acting ceftiofur crystalline-free acid in helmeted guineafowl (Numida meleagris) after a single IM injection. American Journal of Veterinary Research , 72 (11):1514-1518.

Xie W, Zhang $\mathrm{X}$, Wang $\mathrm{T}$ and Du $\mathrm{S}$ (2013).Pharmacokinetic analysis of cefquinome in healthy chickens. Br. Poult . Sci., 54 (1) : 81-86.

Xu J, Fangyuan CAO, Min XU, Yuan ZHU and Yin-sheng QIU (2012).Studies on Pharmacokinetics of Ceftiofur Sodium in Brown Ducks. Chinese J.Vet. Drug, (9): 18-21.

Yancey RJ, Kinney M, Roberts B, Goodenough K, Hamel $\mathrm{J}$ and Ford $\mathrm{C}$ (1987). Ceftiofur Sodium a broad spectrum cephalosporin. Evaluation in vitro in mice. Am. J.Vet. Res., 48 (7) : 1050-1053.

Yuan L, Sun J, Wang R, Sun L, Zhu L, Luo X, Fang B and Liu Y(2011).
Pharmacokinetics and bioavailability of cefquinome in healthy ducks. Am. J. Vet. Res.,72 (1): 122-126. 